\title{
Compton scattering on the nucleon at intermediate energies and polarizabilities in a microscopic model
}

\author{
S. Kondratyuk \\ TRIUMF, 4004 Westbrook Mall, Vancouver, British Columbia, Canada V6T 2A3 \\ O. Scholten \\ Kernfysisch Versneller Instituut, University of Groningen, 9747 AA Groningen, The Netherlands
}

(October 24, 2018)

\begin{abstract}
A microscopic calculation of Compton scattering on the nucleon is presented which encompasses the lowest energies - yielding nucleon polarizabilities - and extends to energies of the order of $600 \mathrm{MeV}$. We have used the covariant "Dressed K-Matrix Model" obeying the symmetry properties which are appropriate in the different energy regimes. In particular, crossing symmetry, gauge invariance and unitarity are satisfied. The extent of violation of analyticity (causality) is used as an expansion parameter.
\end{abstract}

Typeset using REVTEX 


\section{INTRODUCTION}

In this paper we develop a relativistic model suitable for a quantitative description of real Compton $(\gamma N)$ scattering at both low and intermediate energies (up to the second resonance region). To achieve this, the model should obey various constraints that are important in the different energy regimes. At the lowest energies, gauge invariance, CPT invariance and crossing symmetry are important for the model to obey low-energy theorems [1]. At energies near the pion production threshold, unitarity and analyticity put strong constraints on the amplitude [2 7]. Since a wide energy span needs to be described, it is most efficient to use a relativistic approach.

An obvious starting point may seem to be the Bethe-Salpeter equation [8] or a 3dimensional reduction thereof. They have been used to accurately describe pion-nucleon $(\pi N)$ scattering [9 12]. However, with the usual choice of the kernel - consisting of treelevel diagrams only - crossing symmetry is violated. For this reason we have developed an alternative approach, called the "Dressed K-matrix Model" [13 16]. Since this model is based on a K-matrix formalism, unitarity (in the coupled channel space) and crossing symmetry are easily implemented. The kernel is formulated with dressed vertices and propagators such that certain analyticity constraints for the amplitude are incorporated at the level of one-particle reducible diagrams. Singularities of the regularization form factor are chosen far away from the kinematic regime of interest, and thus we ignore the small violations of analyticity due to the regularization. Also, analyticity is not explicitly incorporated for one-particle irreducible diagrams. We argue in Section II that the level of violation of analyticity can be regarded as an expansion parameter in the present model. Gauge invariance is exact through the introduction of contact terms obtained by minimal substitution.

In Section III we present a short recap of the essential ingredients of the Dressed Kmatrix Model. The parameters in the model Lagrangian are fixed to a large extent from pion-nucleon scattering and pion photoproduction as presented in Section [III. The results for Compton scattering and nucleon polarizabilities are given in Section IV]. 


\section{THE DRESSED K-MATRIX MODEL}

Various ingredients of the Dressed K-Matrix Model have been described in Refs. [13]16]. Therefore, here we will present only the essential motivation and arguments behind the formulation of the model. Throughout we will use a manifestly relativistic covariant formulation using the notation of Ref. [22].

Since we want our model to satisfy the important constraint of crossing symmetry, we embed it in the K-matrix approach. In Appendix A we show that any model cast in the K-matrix formalism obeys crossing symmetry if the kernel $K$ itself is crossing symmetric. For a hermitian kernel, unitarity is also satisfied, even in a coupled $\pi$-N and $\gamma$-N channel space. The problem is thus now reduced to constructing a suitable kernel $K$ such that also causality - or analyticity of the amplitude - is satisfied. We will write our kernel as a sum of tree-level diagrams and contact terms (for gauge invariance), as is done usually. If this were

all, the amplitude would not be an analytic function (the real and imaginary parts of the loop corrections would not be related to each other through dispersion relations), which implies that causality would be violated, as is the case in traditional K-matrix models [17 21. The essence of our approach lies in the use of dressed vertices and propagators in the kernel $K$, where the diagrams selected for the dressing are chosen such that certain analyticity constraints are implemented in the calculation of the T-matrix. Effects of the dressing are expressed in terms of purely real form factors and self-energy functions. Our approximation is in the extent to which analyticity of the amplitude for the process is satisfied.

The dressing in our formalism is based on including only those diagrams which are necessary for satisfying analyticity. As discussed in the next section and more extensively in Refs. 13,14, this dressing corresponds to loop corrections to vertices and propagators, where every vertex in a loop correction is in turn corrected by similar loops to satisfy the constraints also in the strong interaction regime. Since this type of dressing cannot be captured as a geometric series in the coupling constant, the summation is done via a numerical iterative expansion. 
In the following we first present the essential ingredients of the dressing procedure for the $\pi N N$ and $\gamma N N$ vertices and the nucleon self-energy and only thereafter show the full structure of the K-matrix kernel.

\section{A. The dressing procedure}

\section{The $\pi N N$ vertex}

The objective of dressing the vertices and propagators in the present approach is solely to improve on the analytic properties of the amplitude. The imaginary parts of the amplitude are generated through the K-matrix formalism (as imposed by unitarity) and correspond to cut loop corrections where the intermediate particles are taken on their mass shell. The real parts have to follow from applying dispersion relations to the imaginary parts. We incorporate these real parts as real vertex and self-energy functions. Investigating this in detail (for a more extensive discussion we refer to [13,14) shows that the dressing can be formulated in terms of coupled equations, schematically shown in Fig. (11), which generate multiple overlapping loop corrections. The coupled nature of the equations is necessary to obey simultaneously unitarity and analyticity.

The equations presented in Fig. (1) are solved by iteration where every iteration step proceeds as follows. The imaginary - or pole - contributions of the loop integrals for both the propagators and the vertices are obtained by applying cutting rules [23]. Since the outgoing nucleon and the pion are on-shell, the only kinematically allowed cuts are those shown in Fig. (1). The real part of the vertex (i.e. the real parts of the form factors) and self-energy functions are calculated at every iteration step by applying dispersion relations 24] to the imaginary parts just calculated, where only the physical one-pion-one-nucleon cut on the real axis in the complex $p^{2}$-plane is considered. These real functions are used to calculate the pole contribution for the next iteration step. This procedure is repeated to obtain a converged solution. We consider irreducible vertices, which means that the external 
propagators are not included in the dressing of the vertices.

One of the advantages of the use of cutting rules in the solution procedure is that throughout we deal with vertices with only one virtual nucleon (half-off-shell vertices). In other words, the knowledge of full-off-shell form factors will not be needed for the calculation of the pole contributions to the loop integrals, which greatly simplifies our approach. Also for the construction of the K-matrix only half-off-shell vertices are required.

In the dressing procedure we maintain the full Lorentz structure of the nucleon propagator and $\pi N N$ vertices. The half-off-shell $\pi N N$ vertex, as given in Appendix $\mathrm{B}$, is written in terms of the form factors $G_{p s}\left(p^{2}\right)$ and $G_{p v}\left(p^{2}\right)$ corresponding to the usual pseudo-scalar and pseudo-vector couplings.

Bare $\pi N N$ form factors

$$
G_{p v}^{0}\left(p^{2}\right)=f(1-\chi) \exp \left[-\ln 2 \frac{\left(p^{2}-m^{2}\right)^{2}}{\Lambda_{N}^{4}}\right] \text { and } G_{p s}^{0}\left(p^{2}\right)=\frac{\chi}{(1-\chi)} G_{p v}^{0}\left(p^{2}\right)
$$

have been introduced in the dressing procedure to regularize the dispersion integrals. Here $\Lambda_{N}^{2}$ is the half-width of the form factor, and $f$ is a bare coupling constant fixed from the condition that the dressed vertex reproduces the physical pion-nucleon coupling on-shell. For simplicity we have taken a vanishing pseudo-scalar admixture $(\chi=0)$ in the bare vertex. The bare form factor reflects physics at energy scales beyond those of the included mesons and which has been left out of the dressing procedure. One thus expects a large width for this factor, as is indeed the case. The use of a bare form factor also implies a violation of analyticity due to additional singularities of this form factor. However, since the width of the form factor is large, the associated violation of analyticity will be small in the energy regime of present interest. It should be noted that our results are largely insensitive to the details of the structure of the bare form factor; only its width matters.

In dressing the $\pi N N$ vertex, the $\Delta, \rho$ and $\sigma$ degrees of freedom are taken into account besides the pion (the vertices and parameters are given in Appendix B and in Table (I)). The coupling parameters were adjusted so that the iteration procedure converges and also to get a reasonable reproduction of pion-nucleon phase shifts in the full model calculation, 
discussed in Section III B. We have insisted on consistency between model parameters in the calculation of the vertices and in the full model calculation. For reasons of simplicity, however, we have not included the full nucleon resonance spectrum in the dressing of the vertices; nor have we dressed all resonance propagators and vertices on the same footing with the nucleon propagator and $\pi N N$ vertex. This can be considered as an additional approximation in the present approach.

The dressed nucleon propagator is renormalized to have a pole with a unit residue at the physical mass and is given in Appendix B, where the nucleon self-energy functions $A\left(p^{2}\right)$ and $B\left(p^{2}\right)$ have been introduced together with the renormalization constant $Z_{2}^{N}$ and the nucleon mass shift $\delta m=m-m_{0}$.

\section{The $\gamma N N$ vertex}

The procedure of obtaining the $\gamma N N$ vertex is in principle the same as for the $\pi N N$ vertex. One should consider the cut loop diagrams generated in the K-matrix approach and use the equivalent cuts for evaluating the integrand of the dispersion integral. This is shown schematically in Fig. (2), a more complete discussion is presented in 16. This equation is solved using the same method as used for constructing the $\pi N N$ vertex. The equation in Fig. (2) is simpler than that in Fig. (四) since, due to the weaker electromagnetic coupling, photon loops are not considered. The dressed $\pi N N$ vertex can thus be taken from the calculations presented in the previous section.

The most general $\gamma N N$ vertex is kept in the dressing procedure. It is given in Appendix B for a real photon with momentum $q=p^{\prime}-p$ and an on-shell outgoing nucleon, $p^{2}=m^{2}$. It contains four form factors $\hat{F}_{1,2}^{+,-}\left(p^{2}\right)$ each of which has the isospin structure $\hat{F}=F^{s}+\tau_{3} F^{v}$.

The bare $\gamma N N$ vertex is taken with $\hat{F}_{1}^{+}\left(p^{2}\right)=\hat{F}_{1}^{-}\left(p^{2}\right)=\hat{e}_{N}=\left(1+\tau_{3}\right) / 2$ and $\hat{F}_{2}^{+}\left(p^{2}\right)=$ $\hat{F}_{2}^{-}\left(p^{2}\right)=\hat{\kappa}_{B}=\kappa_{B}^{s}+\tau_{3} \kappa_{B}^{v}$, the bare anomalous magnetic moment of the nucleon, adjusted to provide the normalization

$$
F_{2}^{+, s}\left(m^{2}\right)=-0.06 \text { and } F_{2}^{+, v}\left(m^{2}\right)=1.85
$$


of the dressed vertex. We have not introduced bare $\gamma N N$ form factors since the dispersion integrals are finite due to the sufficiently fast falloff of the dressed $\pi N N$ vertex.

The contact $\gamma \pi N N$ and $\gamma \gamma N N$ vertices, necessary for gauge invariance of the model, are constructed by minimal substitution in the dressed $\pi N N$ vertex and nucleon propagator, as was explained in [16]. Using minimal substitution, two $\gamma \pi N N$ vertices were derived in [16], which differ by a purely gauge invariant (transverse with respect to the photon fourmomentum) term. In the present calculation, the contact term was chosen as a weighted sum of the two $\gamma \pi N N$ vertices,

$$
\left(\Gamma_{\gamma \pi N N}^{p s}\right)_{\alpha}^{\mu}+\left[0.15\left(\Gamma_{\gamma \pi N N}^{p v 1}\right)_{\alpha}^{\mu}+0.85\left(\Gamma_{\gamma \pi N N}^{p v 2}\right)_{\alpha}^{\mu}\right]
$$

using the notation introduced in Eqs. (C.3,C.5) and (C.6) of Ref. [16]. The reason for this choice will be discussed in Section IIIB, when presenting results for pion photoproduction. Since, due to the inclusion of the $\gamma \pi N N$ contact term, the photon vertex obeys the WardTakahashi identity, the $F_{1}^{ \pm}$form factors are uniquely related to the nucleon self-energy (see Ref. [16]).

As explained in Section $\Pi 1 \mathrm{q}$, the present procedure restores analyticity at the level of one-particle reducible diagrams in the T-matrix. In general, violation due to two- and more-particle reducible diagrams can be regarded as higher order corrections. An important exception to this general rule is formed by one-particle irreducible diagrams generated by the K-matrix formalism for Compton scattering. These include, for example, diagrams where both photons couple to the same intermediate pion in a loop (so-called "handbag" diagrams). In the K-matrix formalism, the imaginary (pole) contribution of this type of diagrams is essentially a square of part of the pion-photoproduction amplitude. This term is exceptional since at the pion threshold the S-wave contribution is large, related to the non-zero value of the $E_{0+}^{1 / 2}$ multipole in pion-photoproduction. Not including the real part of such a large contribution would entail a significant violation of analyticity. To correct for this to a large extent, the $\gamma \gamma N N$ vertex also contains the (purely transverse) "cusp" contact term whose construction is described in Section 4 of Ref. [16]. It is derived from a simplified treatment 
of the large "handbag" loop correction, using the fact that the latter is dominated by the $E_{0+}^{1 / 2}$ photoproduction channel and that therefore the loop can be saturated by $J^{\pi}=1 / 2^{-}$ intermediate states. Since these intermediate states correspond to negative-energy states, the integrand of the dispersion integral for the "cusp" term is proportional to the negativeenergy magnetic (to ensure the transversality of the term) form factor as calculated in the dressing procedure. The explicit expression for the term is given in Eqs. (32) and (33) of Ref. [16], where the functions $\operatorname{Im} \widetilde{F}_{2}^{-}$and $\widetilde{\Sigma}_{I}$ are now obtained from the full dressing procedure.

Since, due to chiral symmetry, the S-wave pion scattering amplitude vanishes at threshold, $\pi \pi N N$ or $\pi \gamma N N$ contact terms analogous to the "cusp" $\gamma \gamma N N$ term will be negligible and have therefore not been considered.

\section{B. The effective Lagrangian}

Since our approach is based on the K-matrix formalism, it is convenient to describe the effective Lagrangian of our model in terms of the diagrams included in the kernel $K$.

The $S$ matrix is expressed in terms of the scattering amplitude $\mathcal{T}$ (the $\mathrm{T}$ matrix) by $S=1+2 i \mathcal{T}$. The T-matrix is written in the form $\mathcal{T}=K+K i \delta \mathcal{T}$, which can be solved, yielding the central equation of the K-matrix approach,

$$
\mathcal{T}=(1-K i \delta)^{-1} K
$$

where $\delta$ indicates that the intermediate particles have to be taken on the mass shell. It is straightforward to check that $S$ is unitary provided $K$ is hermitian. The simplicity of Eq. (4) is due to the fact that it contains the cut two-particle propagator $i \delta$, thus involving integrals only over on-shell intermediate particles. As a result Eq. (4) reduces to a set of algebraic equations when one is working in a partial wave basis. When both the $\pi-N$ and $\gamma-N$

channels are open, the coupled-channel K-matrix becomes a $2 \times 2$ matrix in the channel space, i.e. 


$$
K=\left[\begin{array}{ll}
K_{\gamma \gamma} & K_{\gamma \pi} \\
K_{\pi \gamma} & K_{\pi \pi}
\end{array}\right]
$$

The pion-nucleon scattering entry $K_{\pi \pi}$ of the $\mathrm{K}$ matrix is given in Fig. (3), and the pion photoproduction and Compton scattering entries, $K_{\pi \gamma}$ and $K_{\gamma \gamma}$, are shown in Figs. (4) and (5), respectively, including all baryon resonances specified in Table [1].

The analytic form of the K matrix can be written by applying Feynman rules, with the 3-point vertices and propagators given in Appendix B.

Several coupling constants are fixed from the corresponding decay widths, according to the Particle Data Group values [25]: $g_{\rho^{0} \pi \gamma}=0.131, g_{\rho^{ \pm} \pi \gamma}=0.103$ and $g_{\omega \pi \gamma}=0.313$. The coupling constant $g_{\pi \gamma \gamma}$ is fixed so that the width of the pion decay into two photons is 7.37(1.5) eV. The remaining parameters, given in Table — for the nucleon-meson couplings and in Table 【I for the coupling to the resonances, were determined from a fit to pion-nucleon phase shifts, multipole amplitudes in pion photoproduction and Compton scattering cross sections. The range of the parameters pertaining to the degrees of freedom included in the dressing equations is constrained in addition by the requirement of convergence of the iteration procedure [14.

\section{Violation of analyticity as an expansion parameter}

As we have argued, the amplitude in this model obeys crossing symmetry at the expense of violating analyticity. We have incorporated analyticity constraints at the level of 1-particle reducible diagrams contributing to the amplitude. Analyticity is violated at the level of 2and higher-particle reducible diagrams (the higher-particle reducible diagrams enter when multi-pion production channels are included explicitly). Our approach can thus be regarded as an expansion in a certain parameter $a$, the degree of analyticity of the amplitude, where we interpret the real (principal-value) parts of the dressed one-particle reducible diagrams as terms of $\mathcal{O}(a)$, the real parts of the dressed 1-particle irreducible but 2-particle reducible diagrams as $\sim \mathcal{O}\left(a^{2}\right)$ and so on. Without specifying it, the expansion parameter $a$ is 
related to the energy scale at which the amplitude is affected significantly by imposing the analyticity constraints. By phase-space arguments, the higher-particle reducible diagrams will have finite and comparatively smooth imaginary parts only at higher energies. The associated real parts will thus exhibit an energy dependence which is weaker than those due to the 1- and 2-particle reducible diagrams. In the approach presented here, these weak energy dependences are in general absorbed in one of the fitting constants of the model, such as the strength of the negative energy couplings of the resonances.

We will show in Section $\amalg$ IIA that analyticity at the one-body level (order $\mathcal{O}(a)$ ) strongly affects 3-point vertex functions at relatively moderate momenta. The approach can be extended to include also analyticity at the level of 4-point 2-particle reducible functions. To do so, one will have to add 4-point contact term contributions to the K-matrix. Note that the corresponding imaginary parts are associated with the one-pion-one-nucleon discontinuity. Other diagrams of the orders higher than $\mathcal{O}\left(a^{2}\right)$ will be associated with one-nucleon-multipion thresholds, which implies that they become important above energies of order $m_{N}+$ $2 m_{\pi}$. In other words, in the approximation of two-body unitarity, only the terms of $\mathcal{O}(a)$ and $\mathcal{O}\left(a^{2}\right)$ should be retained. Thus, to systematically improve the property of analyticity of the amplitude, one has to extend the dressing technique to the 1-particle irreducible (but 2-particle reducible) 4-point diagrams. This will ensure that all the terms up to and including $\sim \mathcal{O}\left(a^{2}\right)$ are taken into account. To go beyond second order, one would have to accommodate two-pion production in the model.

In the case of Compton scattering, the "handbag" diagram gives a large contribution with pronounced features at the pion production threshold. Therefore, although this diagram enters at $\mathcal{O}\left(a^{2}\right)$, we had to include it effectively through the "cusp" contact term [16].

An additional breaking of analyticity is caused by the introduction of the bare form factors. Since these are wide, the associated poles in the complex $p^{2}$-plane will be far removed from our region of interest and one may thus regard the associated violation of analyticity being of higher order in $a$. Since a form factor is associated with physics not included explicitly in the model, which in the present case is multi-pion production, one 
could argue that the associated breaking is of the order $\mathcal{O}\left(a^{3}\right)$.

\section{RESULTS}

Results obtained from the dressed K-matrix model are presented in this section. We first discuss effects of the dressing for the vertex functions and nucleon self-energy, showing the large effect of multi-loop dressing. Since these off-shell form factors are not observable quantities, results for pion-nucleon scattering, pion photoproduction and Compton scattering are presented in the following sections.

\section{A. Vertex functions}

As explained above, the dressing of the vertices is expressed in terms of form factors and self-energy functions. They depend of the choice of the bare form factors, but our calculations show that the detailed structure of this bare form factor is rather unimportant, only its half-width $\Lambda_{N}^{2}$ is crucial. There exists a maximum width beyond which the dressing procedure fails to converge. This maximum depends on the meson-nucleon couplings and on the pseudo-scalar - pseudo-vector mixing ratio $(\chi)$ used in the bare form factor. The width we have taken in the calculations discussed in the following, see Table [, has been taken relatively close to this maximum. The form factors for the pion-nucleon vertex are shown in Fig. (6).

Due to the dressing, the pion-nucleon vertex is considerably softer than the original bare vertex. The difference is about a factor one and a half in the width. The dressing affects the pseudo-scalar and pseudo-vector parts of the vertex function differently, resulting in a mixing ratio which is strongly momentum dependent. At the pion-production threshold the ratio is still small, i.e. the pseudo-vector structure dominates, which is consistent with a minor explicit breaking of chiral symmetry.

The nucleon self-energy functions are shown in Fig. (7). The wave function and mass

renormalization constants are $Z_{2}^{N}=0.8$ and $\delta m=-77 \mathrm{MeV}$, respectively. The bare pion 
coupling constant is $f=10.82$ in Eq. (1). In principle, a field redefinition can be made such that the self-energy vanishes, resulting in a transformation of the $\pi N N$ vertex (see [14,15] for details). Due to the equivalence theorem [26], observables calculated in both representations are the same. In the present calculation we have not used a field redefinition.

Since the model is gauge invariant - and thus the $\gamma N N$ vertex obeys the Ward-Takahashi identity - there is a one-to-one correspondence between the electric form factors $F_{1}^{ \pm}$and the nucleon self-energy, given by Eqs. (28) and (29) of Ref. [16]. In particular, the neutronphoton electric form factors are zero. The proton-photon form factors $F_{1}^{ \pm}\left(p^{2}\right)$ are shown in Fig. (8) as functions of the momentum squared of the proton. They do not depend on the choice of the contact $\gamma \pi N N$ vertex.

The magnetic form factors $F_{2}^{ \pm}$are shown in Fig. (9) for the proton and the neutron. The dominant contribution to the form factors $F_{2}^{ \pm}$is due to the first diagram under the integral in Fig. (2), which generates the bulk of the form factors already in the first iteration. This, however, does not mean that the other terms in the equation are of minor importance. In particular, they are crucial for satisfying the Ward-Takahashi identity for the $\gamma N N$ vertex. Because the derivative of the imaginary part of $F_{2}^{-}\left(p^{2}\right)$ tends to infinity as $p^{2}$ approaches the pion threshold (since intermediate s-wave $\pi-N$ states give a large contribution to the loop integrals in Fig. (2)), its real part has a sharp cusp. The dressed vertex is renormalized by adjusting $\hat{\kappa}_{B}\left(\kappa_{B}^{s}=0.017\right.$ and $\left.\kappa_{B}^{v}=1.78\right)$ in the bare $\gamma N N$ vertex to fulfill the normalization conditions Eq. (2).

\section{B. Observables in pion-nucleon scattering and pion photoproduction}

In the following we shall be discussing effects of the dressing of vertices and propagators on observables by comparing two calculations, referred to as calculations B (Bare) and D (Dressed).

- Calculation $B$. The "bare" $\mathrm{K}$ matrix, $K_{B}$, consists of free propagators and bare vertices for all particles. No form factors are included, except for the bare form factors in the 
$\pi N N$ and $\pi N \Delta$ vertices. A $\gamma \gamma N N$ term is not included since the bare $\gamma N N$ vertex does not have form factors. Thus, the corresponding T matrix contains only the pole parts of the loop diagrams.

- Calculation D. The "dressed" K matrix, $K_{D}$, is composed of the dressed propagators for the nucleon, $\Delta, \rho$ and $\sigma$ (the propagators of the pion and $\omega$ are taken free) and the dressed $\pi N N$ and $\gamma N N$ vertices. To provide gauge invariance of the Compton scattering amplitude, a $\gamma \gamma N N$ vertex is added, which includes the additional "cusp" term. Since this calculation includes all features of the model, it is also referred to as the full calculation. Now both pole and principal-value parts are taken into account of a wide class of loop diagrams which contribute to the $\mathrm{T}$ matrix.

Both calculations include a contact $\gamma \pi N N$ vertex, calculated by minimal substitution in the $\pi N N$ vertex (in the bare vertex for calculation B and in the dressed one for calculation D). The $\pi N N$ and $\gamma N N$ vertices are normalized at the threshold to reproduce the physical pion-nucleon coupling constant and the nucleon anomalous magnetic moment, respectively. In addition, nucleon resonances are included in both calculations. Since we focus on effects of the nucleon dressing, we do not readjust the resonance parameters in calculation B.

The pion-coupling parameters have been optimized to reproduce the pion-nucleon scattering phase shifts and inelasticities in the full calculation. However, since in the present paper we do not aim for a perfect fit to the data, we have not used a least-square minimization routine. In part, this is because certain phase shifts, notably the $P_{11}$ at higher energies, show discrepancies which seem to be outside the capability of the model. On the whole, the phase shifts and inelasticities are reproduced well, see Fig. (10). In the propagators for the nucleon resonances an additional width $\Gamma_{0}$ has been introduced, given in Table $\mathbb{1}$, to account for decay channels which are not included explicitly in the model (see Ref. 20] for details). The large effect of the dressing which was seen in the vertex functions persists in the calculation of observables. At pion energies exceeding $500 \mathrm{MeV}$ the phase shift in the $P_{33}$ channel is somewhat above the data. This appears to be due to the structure of the $\pi N \Delta$ 
vertex. Namely, due to the use of a gauge-invariant $\pi N \Delta$ vertex [12], an additional factor $p^{2}$ is introduced in the s-channel diagrams, which is apparently insufficiently compensated by the form factor. We have not made an extensive effort to correct this in the present calculation.

The calculated multipoles for pion photoproduction on the proton are shown in Fig. (11), where the results of both calculations D and B are given. The usual nomenclature [29] for the multipoles is used. Comparing the results of the two calculations in Fig. (11), it is seen that effects of the dressing are most prominent in the magnetic dipole multipole $M_{1-}^{1 / 2}$ reflecting the effect of the $F_{2}^{+}$form factors in the s-channel.

As pointed out earlier, gauge invariance alone does not provide sufficient restrictions on the $\gamma \pi N N$ contact term: its transverse part cannot be determined unambiguously. We found [16] that the choice of the transverse part has an influence on the multipole $E_{0+}^{1 / 2}$, in particular its falloff with energy, which allowed us to fix this term as given in Eq. (3) to fit the data. Since the $E_{0+}^{1 / 2}$ multipole corresponds to angular momentum and parity $J^{\pi}=1 / 2^{-}$ of the nucleon-photon system, it contributes to the imaginary part of $F_{2}^{-}$, which explains the strong effect of the $\gamma \pi N N$ contact term on $F_{2}^{-}$.

\section{COMPTON SCATTERING AND NUCLEON POLARIZABILITIES}

Our special interest concerns observables in Compton scattering since for this case constraints imposed by crossing symmetry and analyticity will be most important.

\section{A. Compton scattering}

The only two free parameters which enter in the calculation of Compton scattering appear in the $\gamma \gamma \sigma$ vertex and have been adjusted to reproduce the backward Compton cross section at moderate energies. Calculated angular distributions are compared with data in Fig. (12). Notably, the near vanishing $0^{\circ}$ cross section at the pion production energy is correctly reproduced in the full calculation, a feature which is impossible to obtain in 
the calculation without dressing. At somewhat higher energies, however, the forward cross section is under-predicted in the full calculation. The dependence of the cross section on the photon energy is displayed in Fig. (13) for different scattering angles. In general the full calculation gives an improvement in the description of the data.

We have not made a detailed investigation of the source of the observed discrepancies with the cross section data. Below the energy of the $\Delta$ resonance the contribution of $\sigma$-meson exchange is small and the most obvious reason for the problems would be the structure of the $\Delta N \gamma$ vertex. At smaller angles there is destructive interference between the $\Delta$ and the nucleon contribution. A relatively minor change in the momentum dependence of the $\Delta N \gamma$ vertex will thus be magnified in its effect on the cross section at forward angles while affecting the larger angles to a lesser extend. For example, the poor agreement at backward angles could be mitigated by lowering the absolute value of the mixing parameter $a_{\gamma}$ in the $\Delta N \gamma$ vertex (see Appendix B and Table II), which would however result in a too high cross section at smaller angles. Above the energy of the $\Delta$ resonance, the contribution of the $\sigma$ meson becomes progressively more important. As a t-channel exchange, it mainly affects the backward angles. Thus, at higher energies and backward angles the cross section will be sensitive to the structure of the vertices in the $\sigma$ exchange diagram. The detailed study of such modifications to the $\Delta$ and $\sigma$ vertices falls, however, outside the scope of the present work.

The photon (beam) asymmetry at $90^{\circ}$ and the proton (target) polarization at $100^{\circ}$ are shown in Fig. (14) as functions of the photon laboratory energy. As can be seen, effects of the dressing become very conspicuous above the $\Delta$ resonance region. The observables exhibit a cusp structure at the pion threshold, which is especially pronounced for the photon asymmetry. This cusp is a consequence of the unitarity and analyticity properties of the coupled-channel scattering matrix, affecting primarily the $f_{E E}^{1-}$ partial wave amplitude [3, 16].

The effect of the dressing on the $f_{E E}^{1-}$ amplitude can be seen in Fig. (15), where also the results of dispersion analyses are quoted for comparison. Note that the imaginary parts of $f_{E E}^{1-}$ from calculations $\mathrm{B}$ and $\mathrm{D}$ are rather similar in the vicinity of threshold. Both 
calculations B and D are unitary, and the full calculation D includes the dressing in addition. Since gauge invariance, crossing and CPT symmetries are fulfilled in the model, all these calculations tend to the Thompson limit at vanishing photon energy.

\section{B. Nucleon polarizabilities}

The polarizabilities characterize response of the nucleon to an externally applied electromagnetic field [35, 36]. They can be defined as coefficients in a low-energy expansion of the cross section or partial amplitudes of Compton scattering. We adhere to the standard notation for the partial amplitudes [2,3]. We will attach the superscript $N B$ (non-Born) to the difference between the full amplitude obtained in the full calculation D and the amplitude in the Born approximation. In the Born approximation, the $\mathrm{T}$ matrix equals the sum of the first two graphs in Fig. (5) with the bare (but properly normalized to the physical anomalous moment) $\gamma N N$ vertices and the free nucleon propagator. Such a calculation is not unitary, resulting in a purely real amplitude. According to the low-energy theorem [1], the zeroth and first orders in an expansion of the amplitude in the small photon energy $\omega$ are model-independent and are reproduced by the Born contribution alone. The polarizabilities enter starting at second order and are model-dependent. We are in particular interested in the role of the dressing procedure in this connection.

To calculate the polarizabilities, we use formulae given in Ref. [6]. The electric and magnetic (scalar) polarizabilities are determined using the equations

$$
\alpha_{E} \simeq \frac{\left(f_{E E}^{1-}+2 f_{E E}^{1+}\right)^{N B}}{\omega^{2}}, \quad \beta_{M} \simeq \frac{\left(f_{M M}^{1-}+2 f_{M M}^{1+}\right)^{N B}}{\omega^{2}}
$$

The spin (vector) polarizabilities are related to third order coefficients in the low-energy expansion,

$$
\begin{gathered}
\gamma_{E 1} \simeq \frac{\left(f_{E E}^{1+}-f_{E E}^{1-}\right)^{N B}}{\omega^{3}}, \quad \gamma_{M 1} \simeq \frac{\left(f_{M M}^{1+}-f_{M M}^{1-}\right)^{N B}}{\omega^{3}} \\
\gamma_{E 2} \simeq \frac{6\left(f_{M E}^{1+}\right)^{N B}}{\omega^{3}}, \quad \gamma_{M 2} \simeq \frac{6\left(f_{E M}^{1+}\right)^{N B}}{\omega^{3}} .
\end{gathered}
$$


We calculate also the forward- and backward-angle spin polarizabilities, given by

$$
\gamma_{0}=-\gamma_{E 1}-\gamma_{M 1}-\gamma_{E 2}-\gamma_{M 2} \quad \text { and } \quad \gamma_{\pi}=-\gamma_{E 1}+\gamma_{M 1}+\gamma_{E 2}-\gamma_{M 2}
$$

respectively. We obtained similar values for the polarizabilities extracted at the energies in the range between $\omega=20 \mathrm{MeV}$ and $\omega=100 \mathrm{MeV}$, whereas at lower energies the numerical extraction was unreliable due to the closeness of the amplitude to the nucleon pole. For this reason, we applied a linear extrapolation to $\omega=0 \mathrm{MeV}$ through the values of polarizabilities calculated at $\omega=80 \mathrm{MeV}$ and $\omega=40 \mathrm{MeV}$ (the points used for the extrapolation are immaterial within the indicated range of moderately low energies).

Since all the parameters are now fixed by the dressing procedure and by the comparison with experiment for the pion-nucleon scattering, pion photoproduction and Compton scattering, the calculated polarizabilities reflect the dynamical contents of the model. Our results for the electric, magnetic and spin polarizabilities of the nucleon are given in Tables $\mathbb{I I I}$ and $[\mathrm{IV}$, where also results of other calculations are summarized together with the values extracted from recent experiments. (It should be noted that there has been some discussion concerning the definition of the polarizabilities used in different chiral perturbation theory calculations, see 41].)

It is known [6] that the t-channel $\pi^{0}$-exchange diagram gives a large contribution to the spin polarizabilities $\gamma$, while not affecting the scalar polarizabilities $\alpha$ and $\beta$. For this reason, this contribution is often subtracted from the $\gamma \mathrm{s}$, as is also done in Tables III and IV. We find that the $\pi^{0}$-exchange diagram gives a contribution of +10.62 to $\gamma_{E 1}^{p}, \gamma_{M 2}^{p}, \gamma_{M 1}^{n}, \gamma_{E 2}^{n}$ and -10.62 to $\gamma_{E 1}^{n}, \gamma_{M 2}^{n}, \gamma_{M 1}^{p}, \gamma_{E 2}^{p}$ (earlier works quote similar numbers: \pm 11.3 [37] \pm 11.2 [4], \pm 10.9 [35], \pm 10.7 [40]). The effect of the dressing on the polarizabilities can be seen by comparing the values given in columns D and B. In particular, the dressing tends to decrease $\alpha$ while increasing $\beta$. Among the spin polarizabilities, $\gamma_{E 1}$ is affected much more than the other $\gamma \mathrm{s}$.

Various contributions to the full calculation D of the polarizabilities are analyzed in Tables $\mathrm{V}$ and $\mathrm{VI}$ for the proton and neutron, respectively. The different rows contain the 
results obtained from the calculation in which certain contributions have been omitted. The $\Delta$-resonance, with its strong magnetic coupling, primarily affect the magnetic polarizabilities such as $\beta, \gamma_{M 1}$ and $\gamma_{M 2}$.

The $\sigma$ meson does not affect the sum of the scalar polarizabilities, $\alpha+\beta$. To understand this feature, we recall that the second order term in the low-energy expansion of the differential cross section in the laboratory frame of reference can be expressed in terms of the polarizabilities as 42

$$
-\frac{m}{2 \alpha_{f}}\left[(\alpha+\beta)(1+\cos \theta)^{2}+(\alpha-\beta)(1-\cos \theta)^{2}\right] \omega^{2}
$$

$\alpha_{f}$ being the fine structure constant, $\alpha_{f}=1 / 137$. The sum $\alpha+\beta$ thus remains unaffected since $\sigma$-exchange enters as a t-channel contribution and does not contribute at forward angles. Also the spin-polarizabilities are not affected by a scalar exchange. Both the $\Delta$ and the $\sigma$ give large, but cancelling, contributions to $\beta$.

The effect of the additional "cusp" $\gamma \gamma N N$ contact term [16], mentioned at the end of Section IIA2, can be seen by comparing the "no cusp" with the full calculation. In particular, it is seen that this term strongly influences the electric polarizabilities rather than the magnetic ones. The reason for this is that the "cusp" contact term affects primarily the

electric partial amplitude $f_{E E}^{1-}$ (corresponding to the the total angular momentum and parity of the intermediate state $\left.J^{\pi}=1 / 2^{-}\right)$rather than the magnetic amplitude $f_{M M}^{1-}\left(J^{\pi}=1 / 2^{+}\right)$. Hence, by Eqs. (6, (6), the electric polarizabilities $\left(\alpha\right.$ and $\left.\gamma_{E 1}\right)$ receive a sizable contribution from this term.

None of the polarizabilities is much influenced by contributions from the $D_{13}$ or any of the other resonances.

\section{SUMMARY AND CONCLUSIONS}

The results are presented of a comprehensive calculation of pion and photon scattering off the nucleon in the "Dressed K-matrix Model". In particular, we focused our attention on 
the calculation of Compton scattering in the energy regime ranging from the lowest energies, where observables are presented in terms of nucleon polarizabilities, up to energies in the second resonance region. We show that this model distinguishes itself from other microscopic approaches since it is able to give a quantitative description of the observables in the full energy range, due to the fact that the model preserves the symmetries which are important in the different energy regimes.

\section{ACKNOWLEDGMENTS}

We would like to thank Alex Korchin for discussions. We also thank Harold Fearing for reading the manuscript and making several useful suggestions. This work is part of the research program of the "Stichting voor Fundamenteel Onderzoek der Materie" (FOM) with financial support from the "Nederlandse Organisatie voor Wetenschappelijk Onderzoek" (NWO).

\section{APPENDIX A: CROSSING SYMMETRY}

The proof that our T-matrix obeys crossing symmetry in the meson lines is based on simple kinematics. Diagrams of the type depicted on the left in Fig. (16) contribute to the T-matrix. Crossing symmetry of the T-matrix implies that also the crossed version of this diagram, depicted on the right in Fig. (16), is taken into account. Part of this diagram corresponds however to the incoming nucleon "decaying" to a state consisting of two on-shell pions and an on-shell nucleon. Since this is not allowed kinematically, the contribution from this crossed diagram vanishes.

This argument can be used to show that for any term contributing to the T-matrix which involves a second or higher power of the kernel, the corresponding crossed diagram vanishes. The resulting T-matrix is thus crossing symmetric provided that the kernel itself is crossing symmetric. In the present approach special care is taken that the latter is indeed the case. 


\section{APPENDIX B: MODEL LAGRANGIAN}

The form factors included in the vertices with baryon and meson resonances have similar form to the bare $\pi N N$ form factor given in Eq. (1), with the half-width chosen the same for all the vertices, $\Lambda^{2}=1 \mathrm{GeV}^{2}$.

The $\Delta N \pi$ and also the $\Delta N \gamma$ vertices have been chosen so as to obey the gauge condition $p \cdot \Gamma=0$ [12]. As a consequence of this the coupling to the spin $1 / 2$ components in the Rarita-Schwinger propagator are eliminated. Only the vertices for the nucleon and the $\Delta$ resonance are given, those for other $j=1 / 2$ and $j=3 / 2$ resonances are similar except for an additional factor $\gamma_{5}$ for resonances carrying negative parity. A cross in the propagator attached to a vertex implies that the particle corresponding to this leg is taken on-shell.

The parameters $a_{\pi}$ and $a_{\gamma}$ appearing in the vertices for spin- $3 / 2$ particles determine the ratio of coupling to positive- and negative-energy intermediate states. In principle the value of the parameter $a_{\gamma}$ can be different for the two structures in the photon-resonance vertex; however, these have been taken the same for simplicity.

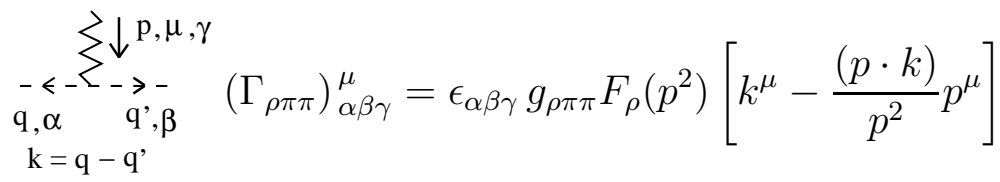

$$
\begin{aligned}
& \vdots_{\vdots} \mathfrak{a}_{-\rightarrow-}\left(\Gamma_{\sigma \pi \pi}\right)_{\alpha \beta}=-i F_{\sigma}\left(p^{2}\right) \delta_{\alpha \beta}\left[g_{\sigma \pi \pi} m_{\pi}+f_{\sigma \pi \pi} \frac{\left(q \cdot q^{\prime}\right)}{m_{\pi}}\right] \\
& \mathrm{q}, \alpha \quad \text { q', } \beta \\
& \vec{\sim} \underset{\sim_{1,--}}{\uparrow \uparrow, \beta}\left(\Gamma_{\rho \pi \gamma}\right)_{\alpha \beta}^{\mu \nu}=-i e \frac{g_{\rho \pi \gamma}}{m_{\pi}} \epsilon^{\mu \nu \rho \sigma} q_{\rho} q_{\sigma}^{\prime} \delta_{\alpha \beta} \\
& \mathrm{q}, \mu \quad \text { q, } \alpha
\end{aligned}
$$

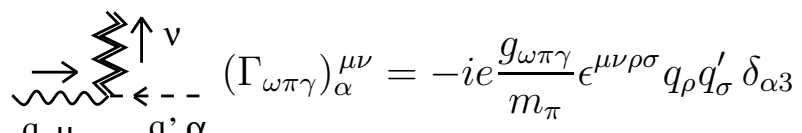

$$
\begin{aligned}
& \text { q, } \mu \quad \text { q', } \alpha \\
& \underset{\mathrm{q}, \mu}{\leftarrow} \sim_{\mathrm{q}, \nu}^{\sim \alpha} \vec{\longrightarrow}\left(\Gamma_{\pi \gamma \gamma}\right)_{\alpha}^{\mu \nu}=-i \frac{e^{2} g_{\pi \gamma \gamma}}{m_{\pi}} \epsilon^{\mu \nu \rho \sigma} q_{\rho} q_{\sigma}^{\prime} \delta_{\alpha 3}
\end{aligned}
$$

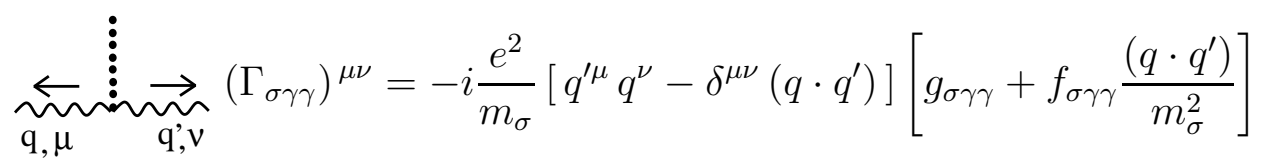




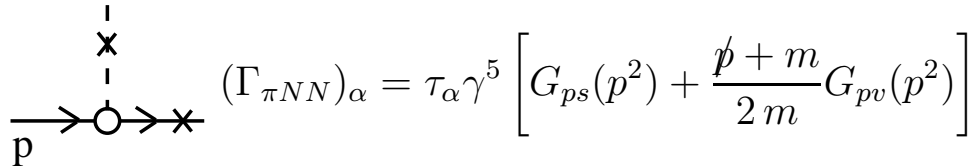

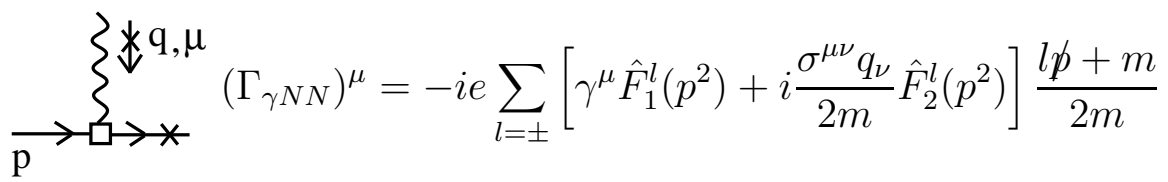

$$
\begin{aligned}
& \underset{\mathrm{p}}{\sum^{\mathrm{k}, \mu, \alpha}}\left(\Gamma_{\rho N N}\right)_{\alpha}^{\mu}=-i g_{\rho N N} F_{N}\left(p^{2}\right) \frac{\tau_{\alpha}}{2}\left(\gamma^{\mu}+i \kappa_{\rho} \frac{\sigma^{\mu \nu} k_{\nu}}{2 m}\right) \\
& \underset{\aleph^{\natural}}{\aleph^{\mathrm{k}, \mu}} \quad\left(\Gamma_{\omega N N}\right)^{\mu}=-i g_{\omega N N}\left(\gamma^{\mu}+i \kappa_{\omega} \frac{\sigma^{\mu \nu} k_{\nu}}{2 m}\right)
\end{aligned}
$$
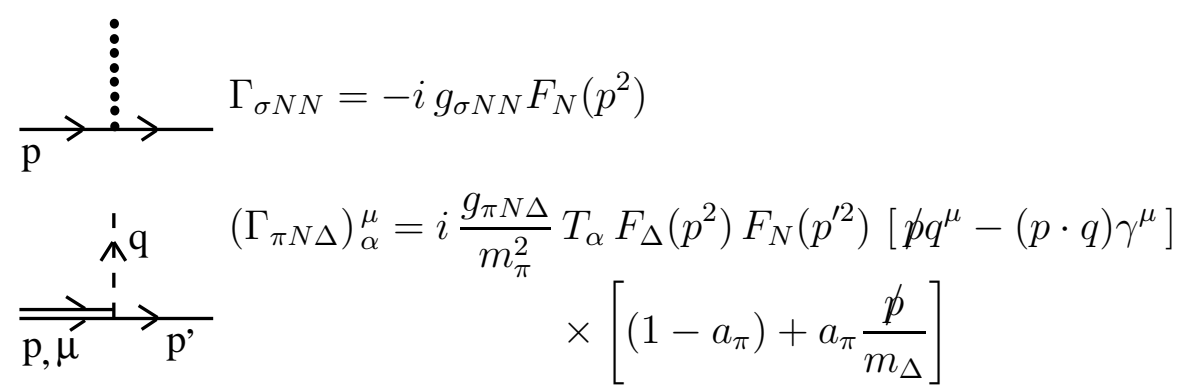

$$
\begin{aligned}
& \left(\Gamma_{\Delta N \gamma}\right)^{\mu \nu}=
\end{aligned}
$$

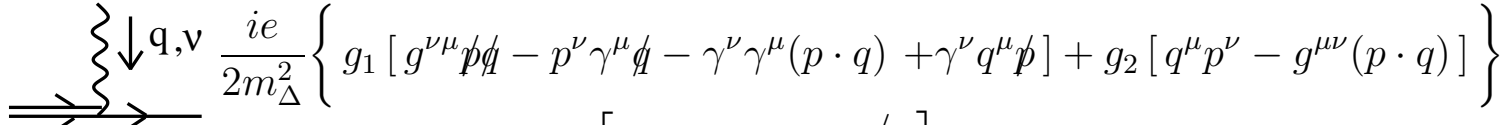

$$
\begin{aligned}
& \stackrel{\longrightarrow}{\mathrm{p}, \mu} \times\left[\left(1-a_{\gamma}\right)+a_{\gamma} \frac{\not p}{m_{\Delta}}\right] \gamma^{5} T_{3}
\end{aligned}
$$

The normalization of the isospin $3 / 2$ to $1 / 2$ transition operators is chosen according to

$$
T_{\alpha} T_{\beta}^{\dagger}=\delta_{\alpha \beta}-\frac{\tau_{\alpha} \tau_{\beta}}{3}
$$

\section{PROPAGATORS}

For cut propagators, the usual form is used as $2 i$ multiplied by the imaginary part of the propagator at positive energies; only the pole contribution is taken into account in the case of the stable particles (nucleon and pion).

- $\mathbf{k}-D_{\pi}^{0}=\frac{i}{k^{2}-m_{\pi}^{2}+i 0}$ 


$$
\begin{aligned}
& \text {....... } D_{\sigma}=\frac{i}{k^{2}-m_{\sigma}^{2}-\Pi_{\sigma}\left(k^{2}\right)} \\
& \Pi_{\sigma}\left(k^{2}\right)=\Pi_{\sigma, L}\left(k^{2}\right)-\left(Z_{\sigma}-1\right)\left(k^{2}-m_{\sigma}^{2}\right)-Z_{\sigma} \delta m_{\sigma}^{2} \\
& \vee^{\mathrm{k}} \sim^{\mu}\left(D_{\rho}\right)^{\mu \nu}=\frac{-i \mathcal{P}_{1}^{\mu \nu}(k)}{k^{2}-m_{\rho}^{2}-\Pi_{\rho}\left(k^{2}\right)}, \mathcal{P}_{1}^{\mu \nu}(k)=g^{\mu \nu}-\frac{k^{\mu} k^{\nu}}{k^{2}}, \\
& \Pi_{\rho}\left(k^{2}\right)=\Pi_{\rho, L}\left(k^{2}\right)-\left(Z_{\rho}-1\right)\left(k^{2}-m_{\rho}^{2}\right)-Z_{\rho} \delta m_{\rho}^{2} \\
& \nu_{\varpi^{\mathrm{k}}}^{\mu} \mu\left(D_{\omega}^{0}\right)^{\mu \nu}=\frac{-i \mathcal{P}_{1}^{\mu \nu}(k)}{k^{2}-m_{\omega}^{2}+i 0} \\
& \stackrel{\mathrm{p}}{\longrightarrow} S_{N}=\frac{i}{\not p-m-\Sigma_{N}(p)+i 0}, \\
& \Sigma_{N}(p)=A_{N}\left(p^{2}\right) \not p+B_{N}\left(p^{2}\right) m-\left(Z_{2}^{N}-1\right)(\not p-m)-Z_{2}^{N} \delta m \\
& { }^{\nu} \stackrel{\mathrm{p}}{\longrightarrow}{ }^{\mu}\left(S_{\Delta}\right)^{\mu \nu}=\frac{-i}{\not p-m_{\Delta}-\Sigma_{\Delta}(p)} \mathcal{P}_{3 / 2}^{\mu \nu}(p), \\
& \Sigma_{\Delta}(p)=A_{\Delta}\left(p^{2}\right) \not p+B_{\Delta}\left(p^{2}\right) m_{\Delta}-\left(Z_{2}^{\Delta}-1\right)\left(\not p-m_{\Delta}\right) \\
& \mathcal{P}_{3 / 2}^{\mu \nu}(p)=g^{\mu \nu}-\frac{1}{3} \gamma^{\mu} \gamma^{\nu}-\frac{1}{3 p^{2}}\left(\not p \gamma^{\mu} p^{\nu}+p^{\mu} \gamma^{\nu} \not p\right)
\end{aligned}
$$

Due to the gauge-invariance condition imposed on the spin-3/2 vertices, the terms in the $\Delta$ propagator proportional to $p^{\mu}$ and $p^{\nu}$ give vanishing contributions to the matrix elements. 


\section{REFERENCES}

[1] F.E. Low, Phys. Rev. 96, 1428 (1954); M. Gell-Mann and M.L. Goldberger, Phys. Rev. 96, 1433 (1954).

[2] W. Pfeil, H. Rollnik, and S. Stankowski, Nucl. Phys. B 73, 166 (1974).

[3] J.C. Bergstrom and E.L. Hallin, Phys. Rev. C 48, 1508 (1993).

[4] A.I. L'vov, V.A. Petrun'kin, and M. Schumacher, Phys. Rev. C 55, 359 (1997).

[5] A. Hünger, J. Peise, A. Robbiano et al., Nucl. Phys. A 62, 385 (1997).

[6] D. Babusci, G. Giordano, A.I. L'vov, G. Matone, and A.M. Nathan, Phys. Rev. C 58, 1013 (1998).

[7] D. Drechsel, M. Gorchtein, B. Pasquini, and M. Vanderhaeghen, Phys. Rev. C 61, $015204(2000)$.

[8] E.F. Salpeter and H.A. Bethe, Phys. Rev. 84, 1232 (1951).

[9] A.D. Lahiff and I.R. Afnan Phys. Rev. C 60, 024608 (1999).

[10] B. C. Pearce and B. K. Jennings, Nucl. Phys. A528, 655 (1991).

[11] F. Gross and Y. Surya, Phys. Rev. C 47, 703 (1993).

[12] V. Pascalutsa and J.A. Tjon, Nucl. Phys. A 631, 534c (1998); Phys. Lett. B 435, 245 (98);Phys. Rev. C 61, 054003 (2000); V. Pascalutsa, Ph.D. thesis, University of Utrecht, 1998.

[13] S. Kondratyuk and O. Scholten, Phys. Rev. C 59, 1070 (1999).

[14] S. Kondratyuk and O. Scholten, Phys. Rev. C 62, 025203 (2000).

[15] S. Kondratyuk and O. Scholten, Nucl. Phys. A 680, 175c (2001).

[16] S. Kondratyuk and O. Scholten, Nucl. Phys. A 677, 396 (2000). 
[17] P. F. A. Goudsmit, H. J. Leisi, E. Matsinos, B. L. Birbrair, and A. B. Gridnev, Nucl. Phys. A575, 673 (1994).

[18] O. Scholten, A.Yu. Korchin, V. Pascalutsa, and D. Van Neck, Phys. Lett. B 384, 13 (1996).

[19] T. Feuster and U. Mosel, Phys. Rev. C 58, 457 (1998).

[20] A. Yu. Korchin, O. Scholten, and R.G.E. Timmermans, Phys. Lett. B 438, 1 (1998).

[21] T. Feuster and U. Mosel, Phys. Rev. C 59, 460 (1999).

[22] J.D. Bjorken and S.D. Drell, Relativistic Quantum Mechanics (McGraw-Hill, 1964); C. Itzykson and J.-B. Zuber, Quantum Field Theory (McGraw-Hill, Inc., 1986).

[23] S. Mandelstam, Phys. Rev. 115, 1741 (1959); R. E. Cutkosky, J. Math. Phys. 1, 429 (1960); G. 't Hooft and M. J. G. Veltman, Diagrammar, CERN Yellow Report 73-09; M. Veltman, Physica 29, 186 (1963).

[24] A. Bincer, Phys. Rev. 118, 855 (1960).

[25] D.E. Groom et. al. , Eur. Phys. J. C15, 1 (2000).

[26] J.S.R. Chisholm, Nucl. Phys. 26, 469 (1961); S. Kamefuchi, L. O’Raifeartaigh, and A. Salam, Nucl. Phys. 28, 529 (1961); H.W. Fearing and S. Scherer, Phys. Rev. C 62, $034003(2000)$.

[27] R.A. Arndt, I.I. Strakovskii, and R.L. Workman, Phys. Rev. C 52, 2120 (1995).

[28] R.A. Arndt, I.I. Strakovskii, and R.L. Workman, Phys. Rev. C 53, 430 (1996).

[29] H. Garcilazo and E. Moya de Guerra, Nucl. Phys. A 562, 521 (1993).

[30] E.L. Hallin et al., Phys. Rev. C 48, 1497 (1993).

[31] G. Galler et al., Phys. Lett. B 503, 245 (2001). 
[32] G. Blanpeid, M. Blecher, A. Caracappa et al., Phys. Rev. Lett. 76, 1023 (1996).

[33] F.V. Adamyan, A.Yu. Buniatian, G.S. Frangulian et al., J. Phys. G: Nucl. Part. Phys. 19 (1993) L139.

[34] Y. Wada, K. Egawa, A. Imanishi et al., Nucl. Phys. B 247, 313 (1984).

[35] T.R. Hemmert, B.R. Holstein, and J. Kambor, Phys. Rev. D 57, 5746 (1998).

[36] B.R. Holstein, hep-ph/0010129.

[37] V. Bernard, N. Kaiser, and Ulf-G. Meissner, Int. J. Mod. Phys. E4, 193 (1995).

[38] V. Bernard, N. Kaiser, A. Schmidt, and Ulf-G. Meissner, Phys. Lett. B 319, 269 (1993).

[39] G.C. Gellas, T.R. Hemmert, and Ulf-G. Meissner, Phys. Rev. Lett. 85, 14 (2000).

[40] K.B. Vijaya Kumar, J.A. McGovern, and M.C. Birse, Phys. Lett. B 479, 167 (2000).

[41] M.C. Birse, X. Ji, and J.A. McGovern, nucl-th/0011054.

[42] I. Guiasu, C. Pomponiu, and E.E. Radescu, Ann. Phys. 114, 296 (1978). 


\section{TABLES}

TABLE I. Meson-nucleon and meson-meson coupling parameters of the model which were optimized to reproduce $\pi N$ scattering phase shifts, pion photoproduction and Compton scattering on the proton. The parameter $\Lambda_{N}^{2}$ is given in $\mathrm{GeV}^{2}$.

$$
\begin{array}{c|cc|cc|ccccc}
\Lambda_{N}^{2} & g_{\rho N N} & \kappa_{\rho} & g_{\omega N N} & \kappa_{\omega} & g_{\sigma N N} & g_{\sigma \pi \pi} & f_{\sigma \pi \pi} & g_{\sigma \gamma \gamma} & f_{\sigma \gamma \gamma} \\
\hline 1.8 & 7.0 & 2.3 & 12 & -0.8 & 34 & 1.7 & 1.8 & -0.42 & -1.7
\end{array}
$$

TABLE II. Parameters for the different $N^{*}$ resonances: masses $(\mathrm{MeV})$, one-pion couplings, two- (and multi-)pion widths (MeV), and off-shell parameters.

\begin{tabular}{c|rr|rr|rrr}
$N^{*}$ resonance & $M_{r}$ & $\Gamma_{0}$ & $g_{\pi N^{*} N}$ & $a_{\pi}$ & $g_{1, \gamma N^{*} N}$ & $g_{2, \gamma N^{*} N}$ & $a_{\gamma}$ \\
\hline$P_{11}(1440)$ & 1550 & 80 & 11.1 & 0.08 & 1.2 & - & - \\
$D_{13}(1520)$ & 1500 & 90 & 1.3 & 0.35 & 4.5 & 5.9 & 0.65 \\
$S_{11}(1535)$ & 1540 & 80 & 1.8 & 1.05 & -1.5 & - & - \\
$S_{11}(1650)$ & 1720 & 100 & 3.9 & 1.05 & -2.2 & - & - \\
$P_{13}(1710)$ & 1720 & 220 & 0.22 & 0.5 & 0 & -3.0 & 0.5 \\
$P_{33}(1232)$ & 1230 & 0 & 2.48 & 0 & -2.33 & -3.02 & -2.40 \\
$S_{31}(1620)$ & 1600 & 30 & 2.25 & 0.75 & -0.20 & - & - \\
$D_{33}(1700)$ & 1650 & 300 & 0.37 & 0.5 & 1.70 & 0 & 0.5 \\
\hline
\end{tabular}


TABLE III. Polarizabilities of the proton. The units are $10^{-4} \mathrm{fm}^{3}$ for $\alpha$ and $\beta$ and $10^{-4} \mathrm{fm}^{4}$ for the $\gamma_{\mathrm{s}}$. The $\gamma_{\mathrm{s}}$ are given without the anomalous $\pi^{0}$ contribution. The first two columns contain the polarizabilities obtained from the present calculation (dressed and bare). The three columns named $\chi P T$ contain the polarizabilities calculated in the chiral perturbation theory: leading order, next-to-leading order and $\mathcal{O}\left(\epsilon^{3}\right)$, from left to right. Results of recent dispersion analyses are given in the last column (Ref. [4] for $\alpha$ and $\beta$ and Ref. [7] for the $\gamma \mathrm{s}$ ).

\begin{tabular}{|c|c|c|c|c|c|c|}
\hline & $\mathrm{D}$ & B & 37 & $\begin{array}{c}\chi \mathrm{PT} \\
38,39(40)\end{array}$ & 35 & $\begin{array}{l}\text { DA } \\
4, \square\end{array}$ \\
\hline$\alpha$ & 12.1 & 15.5 & 12.2 & $10.5 \pm 2.0$ & 16.4 & 11.9 \\
\hline$\beta$ & 2.4 & 1.7 & 1.2 & $3.5 \pm 3.6$ & 9.1 & 1.9 \\
\hline$\gamma_{E 1}$ & -5.0 & -1.7 & -5.7 & $-1.9(-1.3)$ & -5.4 & -4.3 \\
\hline$\gamma_{M 1}$ & 3.4 & 3.8 & -1.1 & $0.4(3.3)$ & 1.4 & 2.9 \\
\hline$\gamma_{E 2}$ & 1.1 & 1.0 & 1.1 & $1.9(1.8)$ & 1.0 & 2.2 \\
\hline$\gamma_{M 2}$ & -1.8 & -2.3 & 1.1 & $0.7(0.2)$ & 1.0 & 0.0 \\
\hline$\gamma_{0}$ & 2.4 & -0.9 & 4.6 & $-1.1(-4.0)$ & 2.0 & -0.8 \\
\hline$\gamma_{\pi}$ & 11.4 & 8.9 & 4.6 & $3.5(6.2)$ & 6.8 & 9.4 \\
\hline
\end{tabular}


TABLE IV. Polarizabilities of the neutron. Explanation of the entries is as in Table III.

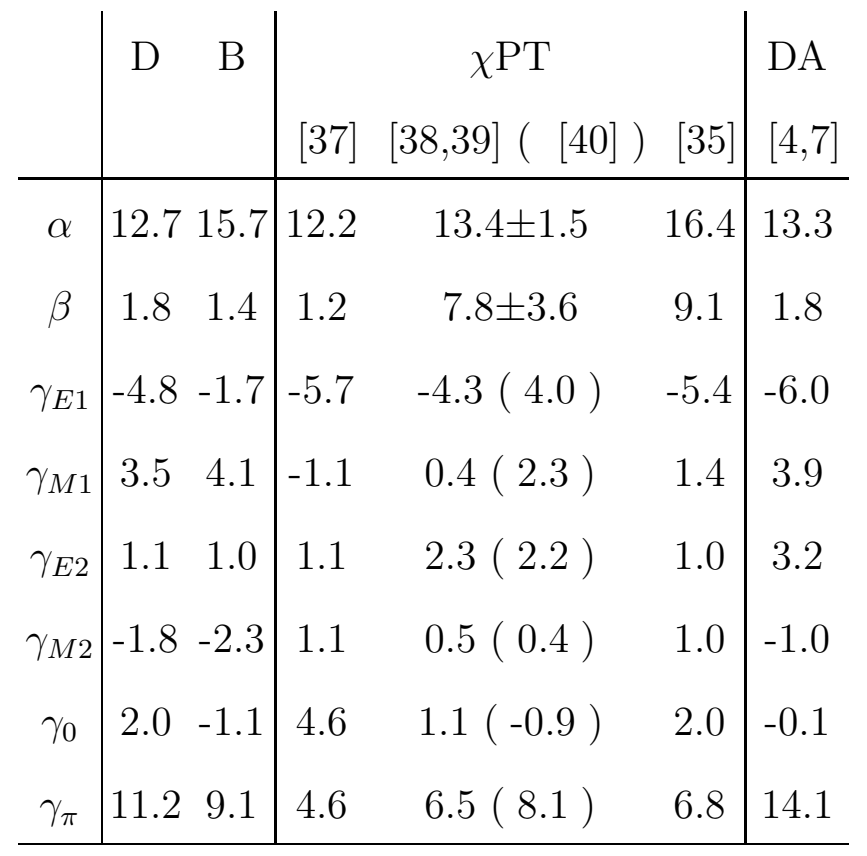

TABLE V. The various contributions to the proton polarizabilities are given. The notation is explained in the text.

\begin{tabular}{r|r|r|r|r|r|r|r|r} 
& $\alpha$ & $\beta$ & $\gamma_{E 1}$ & $\gamma_{M 1}$ & $\gamma_{E 2}$ & $\gamma_{M 2}$ & $\gamma_{0}$ & $\gamma_{\pi}$ \\
\hline Full & 12.1 & 2.4 & -5.0 & 3.4 & 1.1 & -1.8 & 2.4 & 11.4 \\
no $\Delta$ & 13.9 & -11.2 & -3.7 & 0.8 & 0.4 & -0.07 & 2.6 & 4.9 \\
no $\sigma$ & 1.3 & 13.2 & -5.0 & 3.4 & 1.1 & -1.8 & 2.4 & 11.4 \\
no cusp & 8.9 & 2.4 & -1.7 & 3.1 & 0.8 & -1.8 & -0.3 & 7.4 \\
\hline
\end{tabular}

TABLE VI. Same as in Table V, but for the neutron polarizabilities.

\begin{tabular}{r|r|r|r|r|r|r|r|r} 
& $\alpha$ & $\beta$ & $\gamma_{E 1}$ & $\gamma_{M 1}$ & $\gamma_{E 2}$ & $\gamma_{M 2}$ & $\gamma_{0}$ & $\gamma_{\pi}$ \\
\hline Full & 12.7 & 1.8 & -4.8 & 3.5 & 1.1 & -1.8 & 2.0 & 11.2 \\
no $\Delta$ & 14.6 & -11.8 & -3.5 & 0.9 & 0.3 & -0.08 & 2.3 & 4.8 \\
no $\sigma$ & 1.9 & 12.6 & -4.8 & 3.5 & 1.1 & -1.8 & 2.0 & 11.2 \\
no cusp & 9.6 & 1.8 & -1.5 & 3.2 & 0.7 & -1.9 & -0.6 & 7.2 \\
\hline
\end{tabular}




\section{FIGURES}

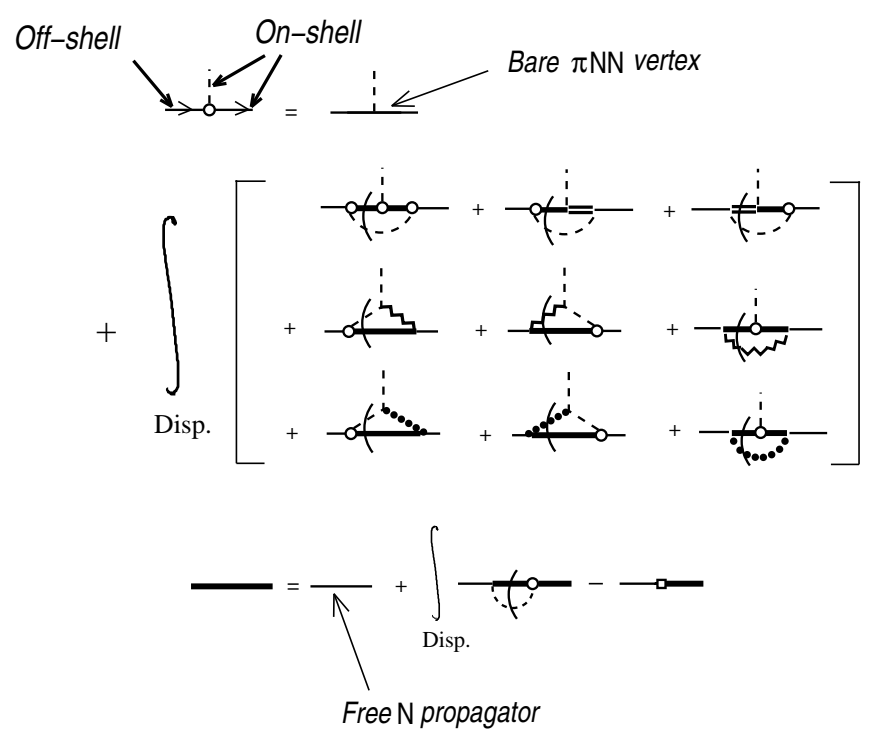

FIG. 1. Graphical representation of the equation for the dressed irreducible $\pi N N$ vertex, denoted by an open circle, and the dressed nucleon propagator, denoted by a solid line. The dashed lines denote pions, the double lines denote $\Delta \mathrm{s}$ and the zigzag and dotted lines are $\rho$ and $\sigma$ mesons, respectively. The resonance propagators are dressed. The last term in the second equation denotes the counter-term contribution to the nucleon propagator, necessary for the renormalization.

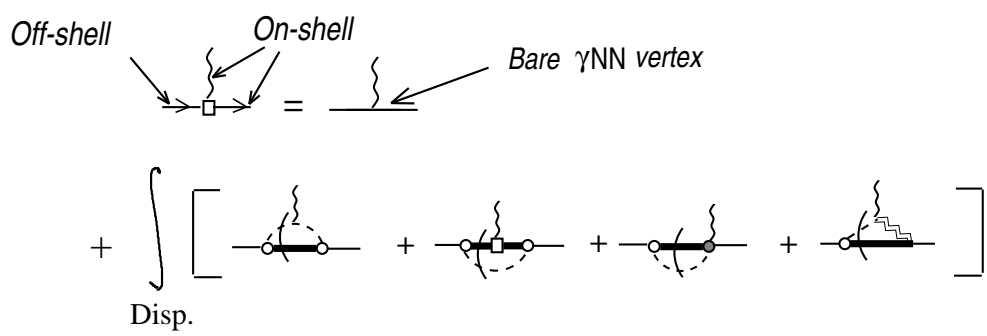

FIG. 2. Equation for the irreducible $\gamma N N$ vertex (denoted by the square) used in the full model. The wiggly lines are photons, the double zigzag line is an $\omega$ meson. See Fig. (1) for an explanation of the other notation. 


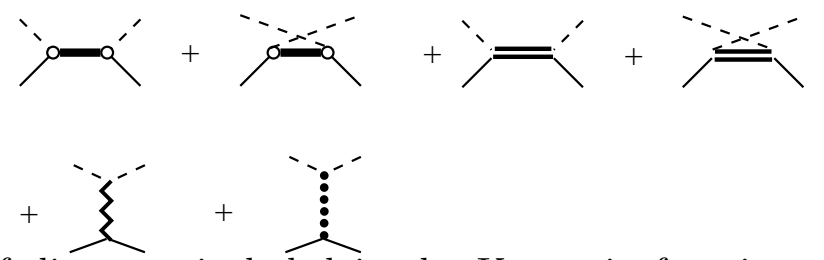

FIG. 3. The sum of diagrams included in the $\mathrm{K}$ matrix for pion-nucleon scattering. The notation is explained in Fig. (11) . The full spectrum of baryon resonances given in Table $\mathbb{1}$ have been included.

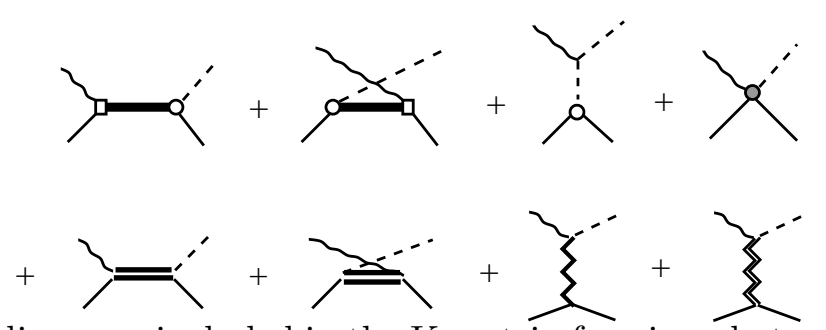

FIG. 4. The sum of diagrams included in the $\mathrm{K}$ matrix for pion photoproduction. The notation is as in Fig. (1) and Fig. (2) The shaded circle is the contact $\gamma \pi N N$ vertex.

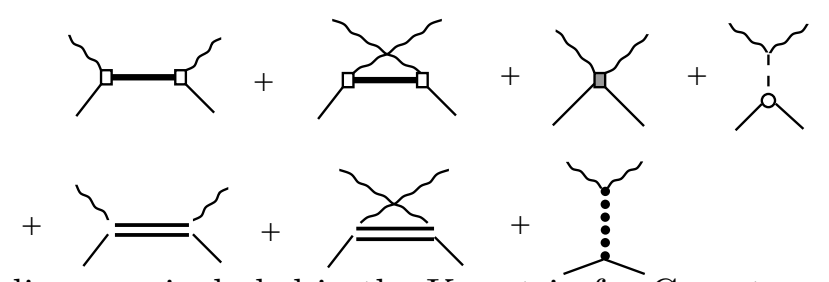

FIG. 5. The sum of diagrams included in the $\mathrm{K}$ matrix for Compton scattering. The notation is as in Fig. (3) and Fig. (㺼). The shaded square is the contact $\gamma \gamma N N$ vertex. 


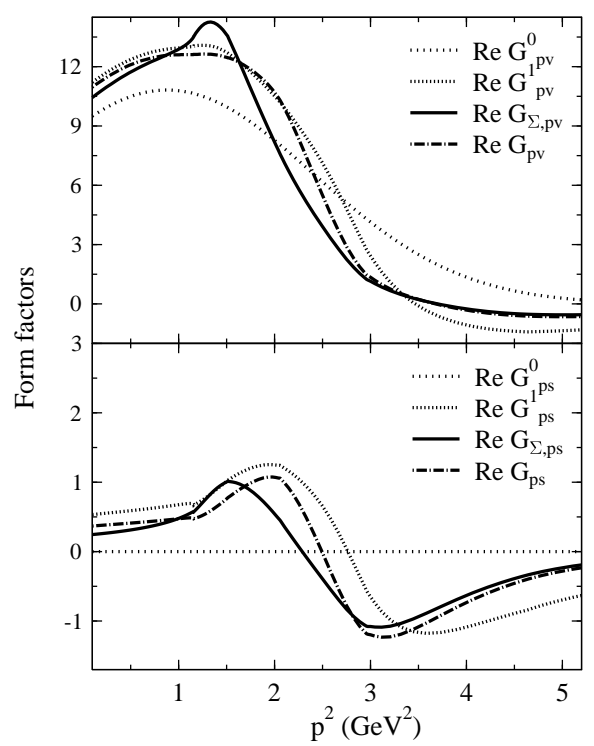

FIG. 6. Dependence of the form factors $G_{p v}$ and $G_{p s}$ entering in the $\pi N N$ vertex, on the momentum squared of the off-shell nucleon. Curves labelled with superscript ${ }^{0}\left({ }^{1}\right)$ show the bare form factor (results of the first iteration). The converged form factors are given in two different representations: one where the nucleon self-energy is non-trivial $\left(G_{\Sigma}\right)$ and one where the self-energy has been transformed out $(G)$.

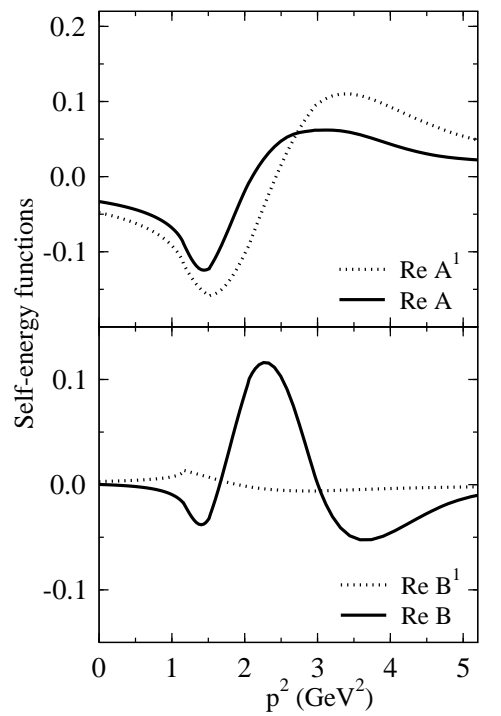

FIG. 7. Dependence of the self-energy functions $A$ and $B$, as enter in the nucleon self-energy, on the momentum squared of the proton. Fully converged results and those from the first iteration are given. 


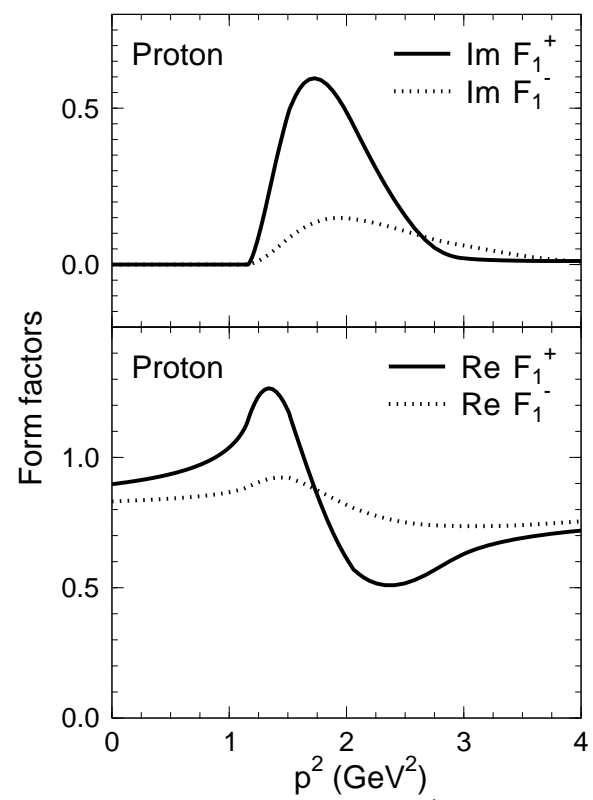

FIG. 8. Dependence of the electric form factors $F_{1}^{ \pm}\left(p^{2}\right)$ on the momentum squared of the off-shell proton.
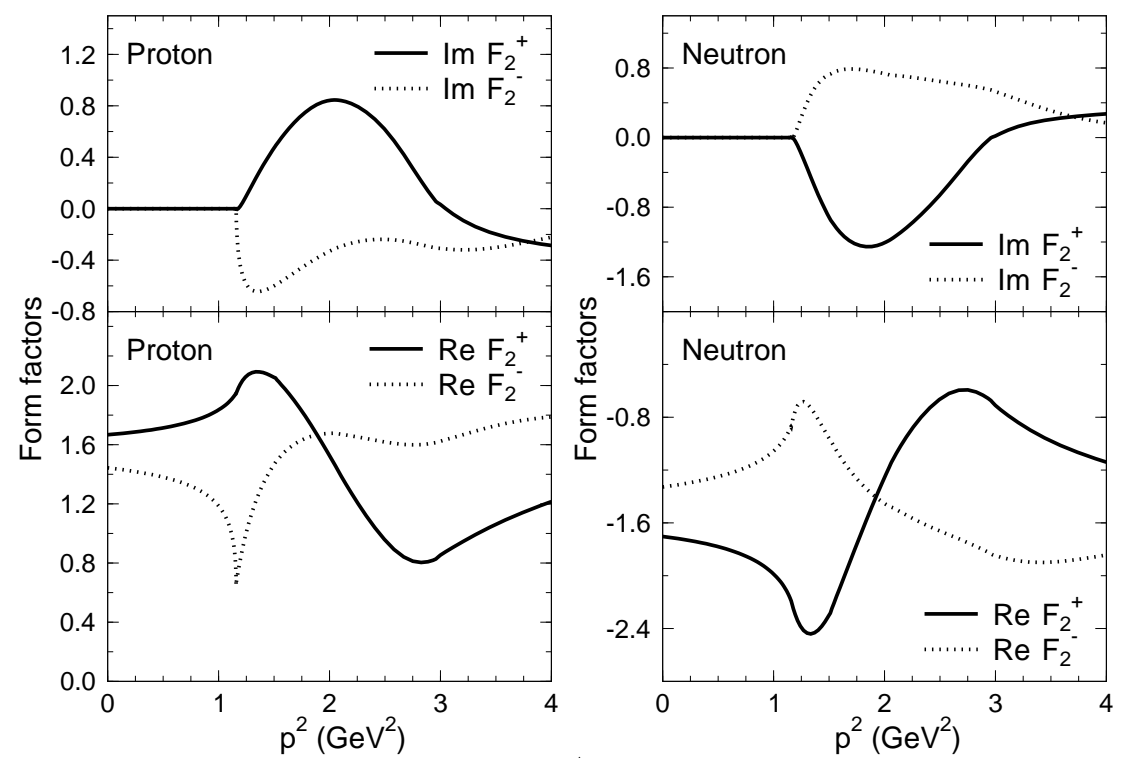

FIG. 9. Magnetic half-off-shell form factors $F_{2}^{ \pm}\left(p^{2}\right)$ for the proton (left panel) and the neutron (right panel). 

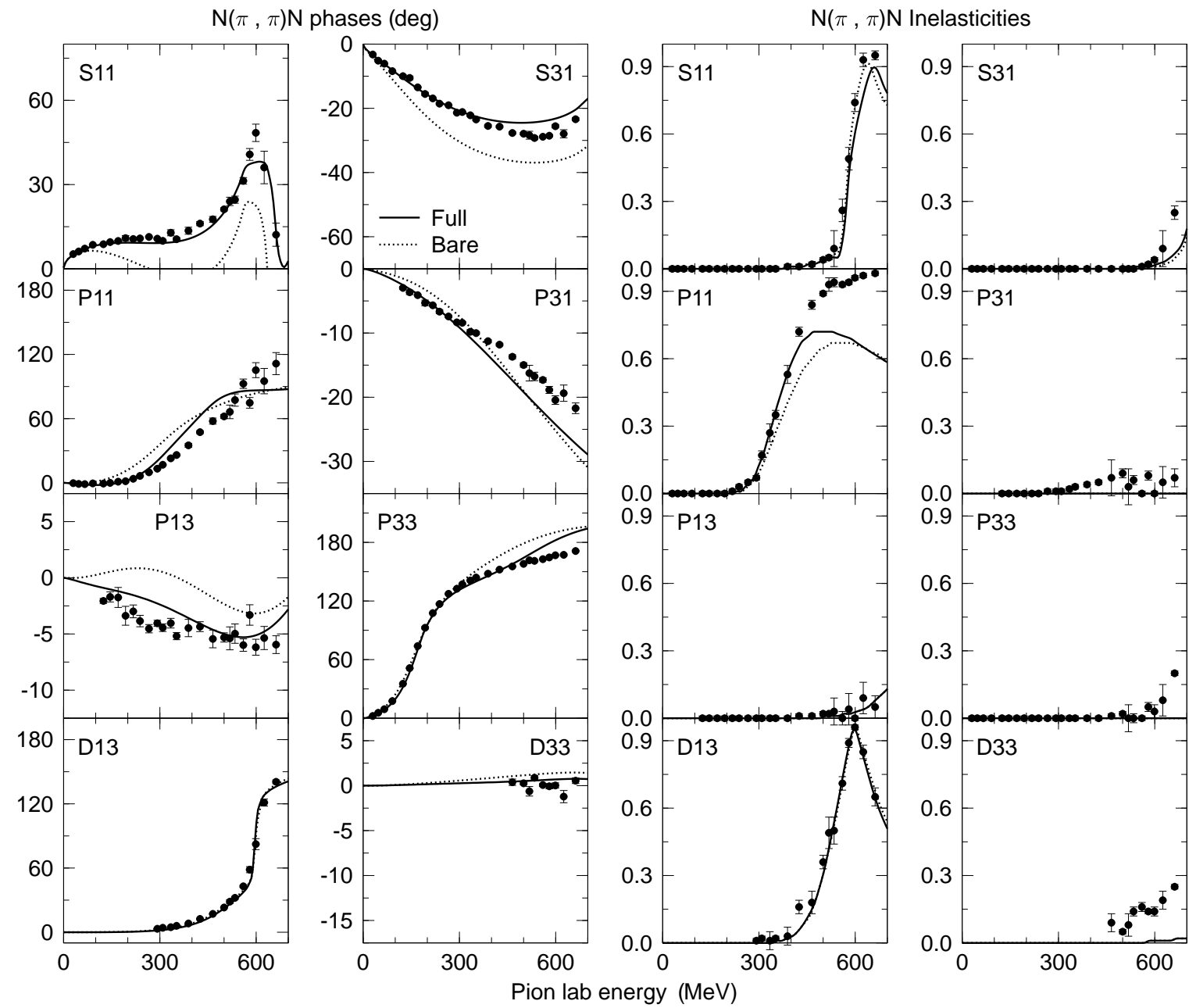

FIG. 10. Phase shifts and inelasticities for pion-nucleon scattering from the analysis of Ref. 27] are compared with the results of the calculations D (full lines) and B (dotted lines). 

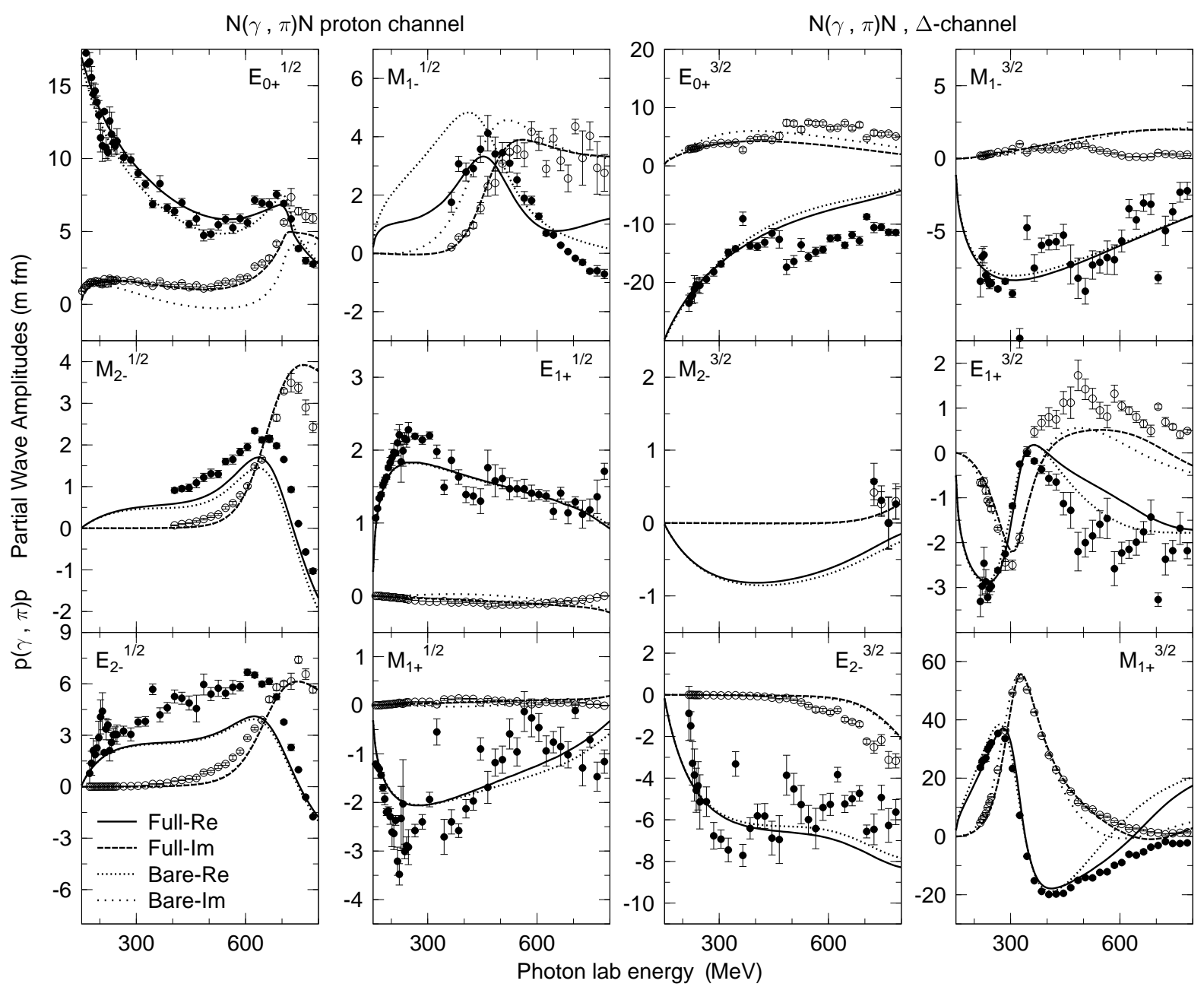

FIG. 11. The calculated pion photoproduction multipoles are compared to the partial-wave analysis results from Ref. [28]. The solid and dashed lines are the real and imaginary parts, respectively, of the multipoles from calculation $\mathrm{D}$. The dense- and sparse-dotted lines are the corresponding quantities obtained without the dressing, i.e. from calculation B. 

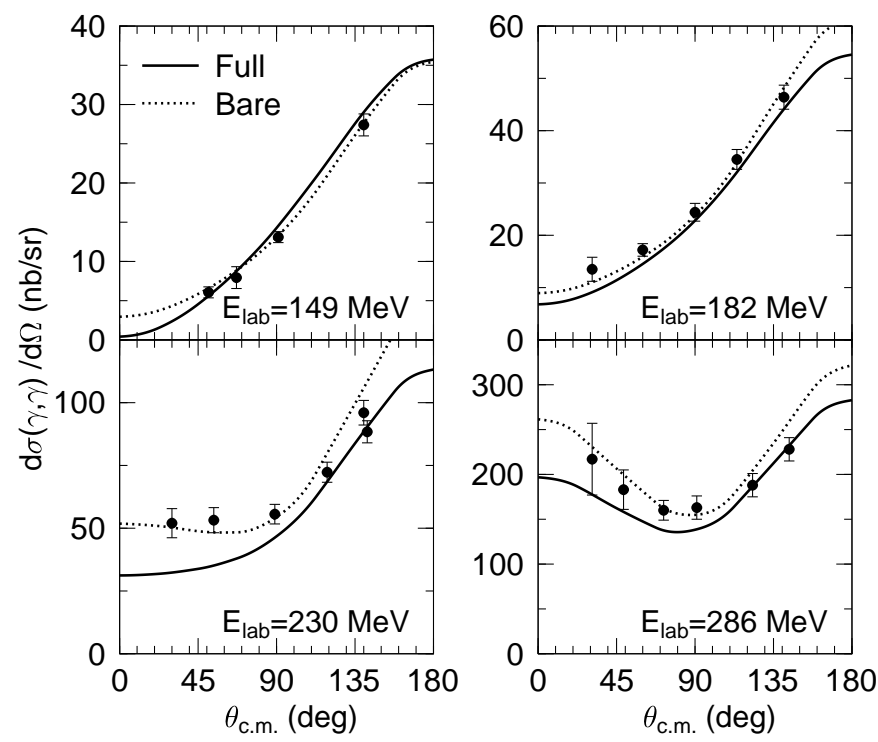

FIG. 12. The angular distributions for Compton scattering are compared with the calculation at different energies. The solid and dotted lines are from the calculations D and B, respectively. The data points are taken from [30]. 


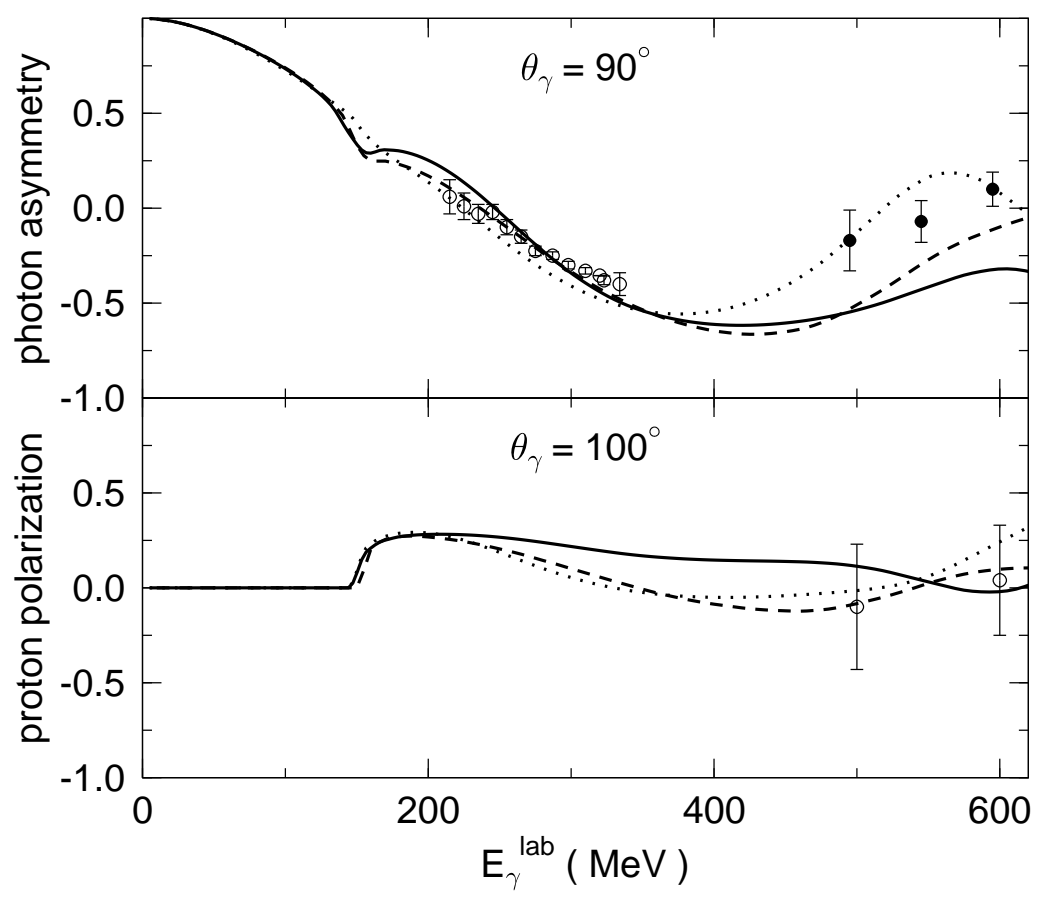

FIG. 14. The photon asymmetry (top) and proton polarization (bottom) as functions the photon laboratory energy, for proton Compton scattering. The solid and dotted lines are from the calculations D and B, respectively. The results of the dispersion calculation from Ref. 沺] are shown by the dashed lines. The data points are taken from the following experiments. For the photon asymmetry: ○ [32], • 33]; for the proton polarization: ○ [34]. 


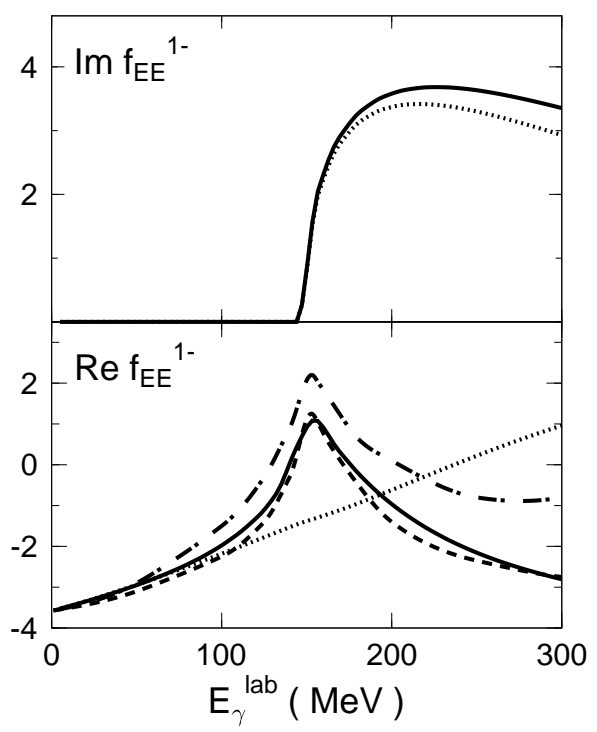

FIG. 15. The $f_{E E}^{1-}$ partial amplitude of Compton scattering on the proton in units $10^{-4} / m_{\pi}$. Solid line: full calculation D; dotted line: calculation B. Also shown are the results of the dispersion analyses of Ref. [2] (dash-dotted line) and Ref. [3] (dashed line).

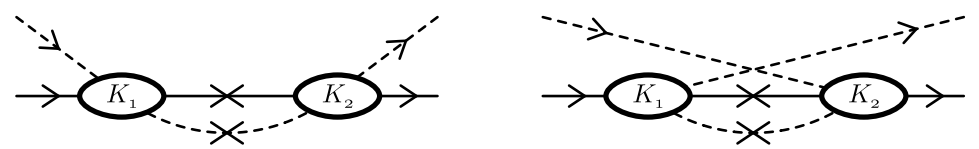

FIG. 16. Left: a diagram contributing to the T-matrix. Pole (on-shell) contributions from propagators are indicated by crosses. Right: the crossed version of the diagram on the left. $K_{1}$ and $K_{2}$ represent results of preceding iterations of the kernel. 